DOI: https://doi.org/10.46296/rc.v5i9.0044

\title{
Acciones de mejora de la inocuidad de los alimentos en el "Gran Hotel" de Camagüey
}

\section{Actions to improve food safety in the "Gran Hotel" in Camagüey

\author{
Cruz-Carbonell Mérida Esmunda
}

Profesora Asistente del departamento de Ciencia y Tecnología de los Alimentos, Universidad de Camagüey Ignacio Agramonte Loynaz. Camagüey, Cuba.

Correo: merida.cruz@reduc.edu.cu

ORCID ID: https://orcid.org/0000-0001-9283-1108

Zambrano-Cedeño Jessica Mariela

Docente investigadora de la Universidad Laica Eloy

Alfaro de Manabí, ULEAM. Manta, Ecuador.

Correo: jessica.zambrano@uleam.edu.ec

ORCID ID: https://orcid.org/0000-0003-1370-5934

\begin{abstract}
Guerra-Vázquez Lizbet
Oficina Territorial de Normalización de Camagüey. Camagüey, Cuba.

Correo: lisazul@gmail.com

ORCID ID: https://orcid.org/0000-0001-6675-8250
\end{abstract}

\begin{abstract}
Agramonte-Corrales Amarilis
Profesora Asistente del departamento de Ciencia y Tecnología de los Alimentos, Universidad de Camagüey Ignacio Agramonte Loynaz. Camagüey, Cuba.

Correo: amarilis.agramonte@reduc.edu.cu

ORCID ID: http://orcid.org/0000-0002-4386-770X
\end{abstract}

\begin{abstract}
RESUMEN
El turismo cultural urbano sustentable es una alternativa para el desarrollo económico, social y cultural de las comunidades, siendo necesario desarrollar servicios turísticos apropiados, como la restauración, prestar atención a la inocuidad de los alimentos, como uno de los cuatro grupos básicos de características junto con las nutricionales, organolépticas y comerciales componen la calidad de un alimento. El objetivo del trabajo es proponer acciones de mejora del servicio de restauración, para la inocuidad de los alimentos en el "Gran Hotel" de Camagüey. Se evaluó el cumplimiento de las buenas prácticas de elaboración de alimentos mediante varias herramientas, como la revisión de documentos de las últimas tres Inspecciones Sanitarias Estatales (ISE) realizadas por el Departamento de Higiene y Epidemiología. Se aplicaron encuestas a los empleados relacionados con el servicio de restauración en las temáticas de higiene y manipulación de alimentos. Se encuestaron clientes externos para conocer el grado de satisfacción con relación al servicio. Fueron identificadas deficiencias higiénico- sanitarias, insatisfacciones de clientes externos y errores de conocimientos básicos para la labor de los manipuladores de alimentos. La propuesta incluye sugerencias prácticas atendiendo a los clientes: internos y externos, de manera que se puedan satisfacer las necesidades y expectativas de ambos segmentos.
\end{abstract}

Palabras claves: servicio de restauración, inocuidad de los alimentos, acciones de mejoras.

Información del manuscrito:

Fecha de recepción: 01 de diciembre de 2021.

Fecha de aceptación: 27 de diciembre de 2021.

Fecha de publicación: 06 de enero de 2022. 


\begin{abstract}
The sustainable urban cultural tourism represents an alternative for the economic, social and cultural development of the urban communities, for that which is necessary to develop appropriate tourist services, inside those that is that of restoration, paying attention prioritized to the inocuidad of the foods that is one of the four basic groups of characteristic together with the nutritional ones, organoleptics and commercial they compose the quality of a nutritious product. The objective of this work is to propose actions of improvement of the restoration service that guarantees the food innocuity in the great hotel of the city of Camagüey. For it, the execution of the good practices of elaboration of foods was evaluated by means of the application of several tools, as the revision of the documentation of the last three sanitary inspections governmentals (ISE, in spanish) carried out by the department of hygiene and epidemiology of the area of health. Surveys were applied the employees related with the restoration service in the thematic of hygiene and manipulation of foods. External clients were interviewed to know the degree of satisfaction with relationship to the restoration service. Hygienic deficiencies were identified - sanitary, external clients' dissatisfactions about the restoration service and errors in the knowledge that are basic for the work that you/they carry out inside the institution the manipulators of foods. The proposal includes practical suggestions assisting the clients: internal and external, so that the necessities and expectations of both segments can be satisfied.
\end{abstract}

Keywords: restoration service, food innocuity, actions of improvements.

\title{
1. INTRODUCCIÓN
}

La abundancia de productos y servicios turísticos en Cuba ejerce presión en las empresas de restaurantes y alojamientos, causando un incremento en el nivel de competitividad e impulsando a las administraciones de estas instalaciones, a aplicar nuevas estrategias que aporten valor a su producto. Una empresa turística no puede limitar su público objetivo a clientes particulares nacionales, cuando existe una gran demanda de clientes y turistas extranjeros. Sin embargo, satisfacer este segmento del mercado implica elevar los estándares de calidad de los servicios y productos ofrecidos. A nivel nacional y mundial, existen diversas instituciones y organismos relacionados a la normativa turística que emiten certificaciones que avalan la conducta, el proceso o los insumos empleados dentro de las empresas y garantizan una experiencia de calidad para el cliente (Soler, 2017).

En publicación realizada en junio de 2019 por la Organización Mundial de la salud, se estima que cada año enferman en el mundo 600 millones de personas por ingerir alimentos contaminados y que 420.000 mueren por la misma causa 
(Organización Mundial de la Salud, 2019), situación que podría prevenirse aplicando las buenas prácticas de manufactura (BPM); principios básicos y prácticos de higiene en la manipulación, preparación, elaboración, envasado, almacenamiento, transporte y distribución de alimentos, con el objeto de garantizar que los productos cumplan con las condiciones sanitarias adecuadas, y disminuir los riesgos de la producción (Niño, 2020).

Todo elaborador, manipulador y consumidor de alimentos tiene la responsabilidad de asegurarse, que estos sean aptos para el consumo. La inocuidad es uno de los cuatro grupos básicos de características que junto con las nutricionales, organolépticas y comerciales componen la calidad de un producto alimenticio. Es la garantía de que este no causará daño al consumidor, cuando sea preparado o ingerido y de acuerdo con el uso a que se destine (NCISO-22000, 2018). En la actualidad y a nivel mundial, la mayor parte de los alimentos llegan al consumidor final, a través de un complejo proceso que se encuentra relacionado a la producción, la manipulación, la elaboración, el almacenamiento, el transporte y la distribución. Un sistema alimentario con un nivel óptimo de eficiencia reduce al mínimo las pérdidas económicas y de recursos, que puedan ser causadas por la manipulación incorrecta, el deterioro o la contaminación de los alimentos. Una gestión eficaz del control de los alimentos constituye un medio importante para mantener la calidad y eficiencia en el sistema alimentario y asegurar la salud de sus clientes (Kotler, 2018).

La inocuidad de los alimentos es un elemento fundamental de la salud pública y un factor determinante del comercio de alimentos; involucra varias personas interesadas, entre ellos productores primarios, manipuladores de alimentos, los elaboradores y comerciantes, a lo largo de toda la cadena alimenticia, los servicios de expendio de alimentos y los consumidores (Ferreiro, 2019).

Para la competitividad en la cadena alimentaria, la inocuidad y salubridad son como ejes fundamentales. Tener en cuenta el nuevo paradigma de consumo de agroalimentos, es importante para adaptar la forma en que los producimos, garantizando altos estándares de calidad y sostenibilidad (Bergaglio, 2018).

Cuando una institución turística logra desarrollar una filosofía de calidad desarrolla una concepción integral que anima a la mejora continua y logra que 
todos sus miembros se involucren, su centro de atención será la satisfacción tanto del cliente. Lo anterior significa que se logra que los todos los trabajadores estén completamente comprometidos e involucrados con la calidad, que los requerimientos del cliente son percibidos y tomados integralmente.

Debido a lo señalado anteriormente, este estudio se relaciona con el diseño de un plan de mejora, con la finalidad de aumentar la calidad en los servicios de restauración que brinda el Gran Hotel y de ajustar sus procesos a un mejoramiento continuo que le permita mantener la inocuidad alimentaria y alcanzar sus metas funcionales de forma efectiva y confiable.

Cuba aspira a ofrecer servicios competitivos en cuanto a calidad e higiene en la restauración, por lo que se encuentra enfrascada actualmente en recuperar el sistema turístico y posicionarse en el mercado mundial para restablecer la comercialización existente en el país hasta marzo del 2020. Durante inspecciones sanitarias realizadas se detectaron, en este hotel, deficiencias en las Inspecciones Sanitarias Estatales (ISE) por Especialistas de Higiene y Epidemiologia, Ministerio del turismo, donde se evidencia la falta de certificación actual de $\mathrm{C}_{+} \mathrm{HS}$, e insuficiente capacitación del personal de cocina, en los procesos de inocuidad y las nuevas tendencias del 2022 .En correspondencia con los aspectos antes señalados, la investigación se propone como objetivo de desarrollar acciones de mejora del servicio de restauración, que garantice la inocuidad de los alimentos en el "Gran Hotel" de la Ciudad de Camagüey.

\section{METODOLOGÍA}

El presente trabajo se realizó en el Gran Hotel de la Ciudad de Camagüey, durante el período comprendido entre los meses de enero a marzo del 2020.

La medición, como método empírico, se utilizó para comparar los patrones seleccionados a partir de encuestas realizadas a los clientes internos y externos para analizar su criterio y valoración sobre la calidad del servicio de restauración de la instalación turística.

Como método teórico, la deducción posibilitó la aplicación de las cuestiones generales conocidas sobre el tema de la inocuidad de los alimentos en la instalación que fueron objeto de las técnicas de investigación, a partir del 
establecimiento de un enlace de juicios que guiaron el cuestionario contenido en cada uno de los instrumentos utilizados.

\section{Instrumentos de recolección de datos:}

Se utilizaron como instrumentos para esta investigación los siguientes:

1. Cuestionario a clientes externos: Fueron encuestados 285 clientes, con preguntas mixtas, para indagar sobre su percepción acerca de la calidad de la restauración del hotel en la ciudad de Camagüey.

2. La encuesta fue aplicada al personal del hotel, 59 trabajadores (clientes internos). Compuesta por preguntas cerradas relacionadas con aspectos vinculados con la manipulación de los alimentos y las Buenas Prácticas de Manufactura.

3. La encuesta que se realizó a 285 clientes externos: Integrada por preguntas cerradas relacionadas con los aspectos desglosados en relación con el servicio de restauración.

4.Revisión de la documentación de las Inspecciones Sanitarias Estatales (ISE) realizadas por el área de salud (las tres últimas realizadas).

5.Revisión documental: vinculada a revisión de los documentos relacionados con la temática a investigar, que brindarán los datos necesarios para el diagnóstico y posterior propuesta de mejora.

Mediante la investigación se podrá describir el nivel de satisfacción con la atención según la percepción de los clientes internos y externos sobre la calidad del servicio de restauración especialmente sobre la inocuidad de los alimentos, que brinda la instalación hotelera.

Además, se realizó el estudio bibliográfico mediante libros, revistas, informes de tesis, informes de la institución, referidos al tema de la investigación, unido al material digital existente en bibliotecas y Scholar Google. Con fines organizativos se emplearán las fichas bibliográficas para anotar los datos de los libros y demás documentos consultados durante la investigación. 


\section{ANÁLISIS E INTERPRETACIÓN DE LOS RESULTADOS}

\subsection{Encuesta 1}

Se encuestaron 59 clientes internos, con una escala ascendente respecto a la calidad, con valores de 1 a 5 atendiendo a los aspectos siguientes:

Tabla 1. Resultados de la encuesta a 59 clientes internos.

\begin{tabular}{|c|c|c|c|c|c|}
\hline 1. Área de trabajo & 1 & 2 & 3 & 4 & 5 \\
\hline 1. Organización y limpieza del trabajo en el área. & & & 26 & 25 & 8 \\
\hline $\begin{array}{l}\text { 2. Definición y conocimiento de sus funciones } \\
\text { responsabilidades. }\end{array}$ & & & 20 & 25 & 14 \\
\hline 3. Distribución equitativa de cargas de trabajo. & & & 21 & 30 & 8 \\
\hline 4. Disponibilidad de sustancias químicas para la limpieza. & & & 10 & 41 & 7 \\
\hline 5. Momentos en los que se realiza la limpieza del área. & & & 9 & 43 & 7 \\
\hline \multicolumn{6}{|l|}{ 2. Dirección del área } \\
\hline $\begin{array}{l}\text { 1. Conocimiento y dominio de funciones por parte del } \\
\text { responsable del área. }\end{array}$ & & & 20 & 26 & 13 \\
\hline $\begin{array}{l}\text { 2. Resolución eficaz de problemas por parte del responsable del } \\
\text { área. }\end{array}$ & & & 25 & 20 & 14 \\
\hline $\begin{array}{l}\text { 3. Delegación eficaz de funciones de responsabilidad por parte } \\
\text { del responsable del área. }\end{array}$ & & & 23 & 29 & 7 \\
\hline $\begin{array}{l}\text { 4. Inclusión y participación del personal en la toma de decisiones } \\
\text { del área. }\end{array}$ & & & 20 & 25 & 14 \\
\hline $\begin{array}{l}\text { 5. Retroalimentación del responsable del área, sobre aspectos } \\
\text { que afecten su desempeño. }\end{array}$ & & & 33 & 15 & 11 \\
\hline \multicolumn{6}{|l|}{ 3. Servicio de restauración } \\
\hline 1. La restauración atendida con mayor prioridad en este servicio. & & & 19 & 25 & 15 \\
\hline 2. Prioridad al proceso de elaboración de los alimentos. & & & 21 & 30 & 8 \\
\hline $\begin{array}{l}\text { 3. Preservación de las características organolépticas y nutritivas } \\
\text { de los alimentos. }\end{array}$ & & & 23 & 29 & 7 \\
\hline 4. Presentación y decoración de los platos. & & & 10 & 43 & 4 \\
\hline 5. Limpieza de equipos y utensilios. & & & 10 & 30 & 19 \\
\hline \multicolumn{6}{|l|}{ 4. Almacenamiento de alimentos } \\
\hline 1. Estado de los almacenes. & & & 25 & 20 & 14 \\
\hline $\begin{array}{l}\text { 2. Estado de las cámaras de frio y equipos de medición de la } \\
\text { temperatura }\end{array}$ & & & 19 & 30 & 10 \\
\hline 3. Descongelación de los alimentos. & & & 23 & 25 & 8 \\
\hline 4. Recolección y disposición de los desechos. & & & 31 & 18 & 10 \\
\hline 5. Limpieza del almacén, cocina y otras áreas aledañas. & & & 23 & 29 & 7 \\
\hline \multicolumn{6}{|l|}{ 5. Condiciones laborales } \\
\hline 1. Seguridad laboral del puesto de trabajo. & & & 21 & 30 & 8 \\
\hline
\end{tabular}


Revista Científica Arbitrada de Investigación en Comunicación, Marketing y Empresa REICOMUNICAR. Vol. 5, Núm. 9 (ene - jun 2022) ISSN: 2737-6354.

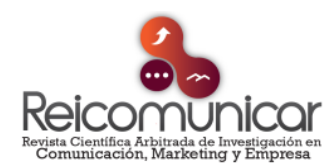

\begin{tabular}{|c|c|c|c|}
\hline $\begin{array}{l}\text { 2. Las condiciones ambientales para desarrollar actividades } \\
\text { diarias. }\end{array}$ & 20 & 32 & 7 \\
\hline $\begin{array}{l}\text { 3. Infraestructura e instalaciones del área para el desarrollo del } \\
\text { trabajo. }\end{array}$ & 10 & 40 & 9 \\
\hline 4. Equipos y herramientas suficientes y acordes a su trabajo. & 8 & 42 & 9 \\
\hline \multicolumn{4}{|l|}{ 6. Capacitación y formación } \\
\hline $\begin{array}{l}\text { 1. Capacitación necesaria para desempeñar correctamente su } \\
\text { trabajo. }\end{array}$ & 20 & 30 & 9 \\
\hline $\begin{array}{l}\text { 2. Atención de necesidades personales con respecto a } \\
\text { capacitación. }\end{array}$ & 21 & 30 & 8 \\
\hline $\begin{array}{l}\text { 3. Atención a necesidades profesionales con respecto a } \\
\text { capacitación. }\end{array}$ & 21 & 30 & 8 \\
\hline 4. Contenido suficiente de las capacitaciones. & 18 & 31 & 10 \\
\hline 5. Frecuencia de la aplicación de capacitaciones. & 31 & 18 & 10 \\
\hline \multicolumn{4}{|l|}{ 7. Implicación en la mejora } \\
\hline 1. Conocimiento de los objetivos del área. & 20 & 30 & 9 \\
\hline $\begin{array}{l}\text { 2. Proposición y puesta en marcha de iniciativas de mejora en los } \\
\text { procesos del área. }\end{array}$ & 20 & 30 & 9 \\
\hline $\begin{array}{l}\text { 3. Proposición y puesta en marcha de iniciativas de mejora en la } \\
\text { calidad de los servicios. }\end{array}$ & 22 & 28 & 9 \\
\hline $\begin{array}{l}\text { 4. Identificación clara de aspectos susceptibles de mejora en el } \\
\text { área. }\end{array}$ & 20 & 32 & 7 \\
\hline $\begin{array}{l}\text { 5. Tendencia de mejoría en la calidad del servicio, en el último } \\
\text { año. }\end{array}$ & 10 & 40 & 9 \\
\hline \multicolumn{4}{|l|}{ 8. Motivación y reconocimiento } \\
\hline 1.Coparticipación en los éxitos y fracasos de su área de trabajo & 22 & 30 & 7 \\
\hline 2. Motivación y gusto por el trabajo que desarrolla. & 7 & 18 & 34 \\
\hline 3. Reconocimiento adecuado de las tareas que desarrolla. & 10 & 39 & 10 \\
\hline 4. Reconocimiento especial por mejoras en su trabajo. & 10 & 39 & 10 \\
\hline 5. Conformidad con el cargo que desempeña actualmente. & 7 & 18 & 34 \\
\hline
\end{tabular}

En las respuestas obtenidas de los clientes internos del hotel, los indicadores evaluados se ubican por lo general en valores entre 3 y 4 , lo que demuestra que en todos se puede incidir para lograr elevar el nivel de conocimiento y desempeño en el trabajo, aspectos que a su vez constituyen la motivación necesaria para brindar una mejor atención a los clientes externos que visitan la instalación. Los valores más bajos están relacionados con la recolección y disposición de los desechos, la retroalimentación del responsable del área sobre aspectos que afecten su desempeño y la frecuencia de la aplicación de 
capacitaciones; problemas que son de fácil y rápida solución, ya que no requieren de grandes esfuerzos y recursos, pero que deben ser atendidos oportunamente pues pueden incidir de manera directa en la calidad de las atenciones que presta la instalación y afectarse el servicio de restauración.

\subsection{Resultado de la encuesta a trabajadores del hotel}

Esta encuesta fue aplicada a 36 trabajadores del hotel vinculados directamente con el servicio de restauración y que se necesita conocer el nivel de conocimientos sobre aspectos que puedan influir en la inocuidad de los alimentos.

Tabla 2. Resultados de la encuesta a clientes internos.

\begin{tabular}{|l|c|c|c|c|c|c|c|}
\hline Preguntas & ítem 1 & ítem 2 & ítem 3 & Preguntas & ítem 1 & ítem 2 & ítem 3 \\
\hline $\mathbf{1}$ & 94.4 & 5.5 & - & 9 & 100 & & \\
\hline 2 & 91.6 & 8.33 & 0 & 10 & 94.4 & 5.5 & \\
\hline 3 & & & 100 & 11 & 8.33 & & 91.6 \\
\hline 4 & & & 100 & 12 & 5.5 & 94.4 & \\
\hline 5 & 5.5 & 94.4 & & 13 & & 100 & \\
\hline 6 & & & 100 & 14 & 94.4 & 5.5 & \\
\hline 7 & 5.5 & 94.4 & & 15 & 5.5 & 94.4 & \\
\hline 8 & 91.6 & & 8.33 & & & & \\
\hline
\end{tabular}

Como resultado del procesamiento de esta encuesta se observa que existen trabajadores que tienen dificultades en algunos conocimientos que son básicos para la labor que realizan dentro de la institución, aspectos relacionados con la conceptualización de manipulador de alimentos y con las partes de su cuerpo que deben mantener bien higienizadas durante su trabajo.

El manipulador de alimentos es un trabajador que ha de tener los hábitos higiénicos más estrictos que otros trabajadores, debido a la gran responsabilidad de crear alimentos inocuos. Por ello debe adoptar un sistema de autocontrol higiénico en su trabajo y conocer el proceso de preparación y conservación de alimentos, respetando las exigencias culinarias, sanitarias y nutritivas que permitan que el alimento llegue al consumidor en las mejores condiciones de calidad evitando que en muchos casos sea el manipulador el que interviene 
como vehículo de transmisión en la contaminación de alimentos, por actuaciones incorrectas y malas prácticas higiénicas.

Por otra parte, se expresó por algunos de los encuestados que usan el uniforme fuera de la instalación, es decir que es usado para diversos fines, esta práctica es muy riesgosa pues en el vestuario puede recoger un número elevado de microorganismos; esta microbiota y todo lo que entra en contacto con ella puede causar varios tipos de enfermedades alimentarias, las bacterias que llegan al vestuario pueden multiplicarse en este, especialmente en las dobleces, costuras y bolsillos y ser transportados a diferentes lugares.

Algunos trabajadores seleccionan acudir al médico solo en caso de enfermedad, es decir desconocen la importancia del chequeo médico periódico que deben realizarse como acciones importantes por su vinculación directa con los alimentos, ya que existen personas asintomáticas de diferentes enfermedades y los microrganismos pueden contaminar los alimentos.

Existen encuestados que manifiesta utilizar un apósito o guantes cuando presentan una herida. Por el contrario, deben retirarse del servicio, no deben manipular alimentos, puesto que las heridas y cortes en las manos constituyen focos de infección y pueden contaminar los alimentos, así como que los alimentos crudos y cocidos pueden almacenarse juntos siempre que estén refrigerados, acción riesgosa pues puede originar contaminación cruzada.

También, manifiesta una minoría que, al utilizar tablas diferentes durante la preparación de alimentos se evita la contaminación de olores, sin hacer mención a la contaminación cruzada que es un riesgo frecuente que se corre con esa acción, además, algunos marcaron que no tienen conocimientos de lo que es una ETA, aspectos fundamentales que deben conocer los manipuladores de alimentos y que deben ser abordados en breve durante el proceso de capacitación.

\subsection{Resultados de la encuesta a clientes externos}

La encuesta aplicada a los clientes externos, una vez tabulados sus resultados, atendiendo a cada una de las preguntas formuladas, se obtuvo el siguiente resultado: 
Figura 1. Percepción sobre la calidad de los desayunos del "Gran Hotel".

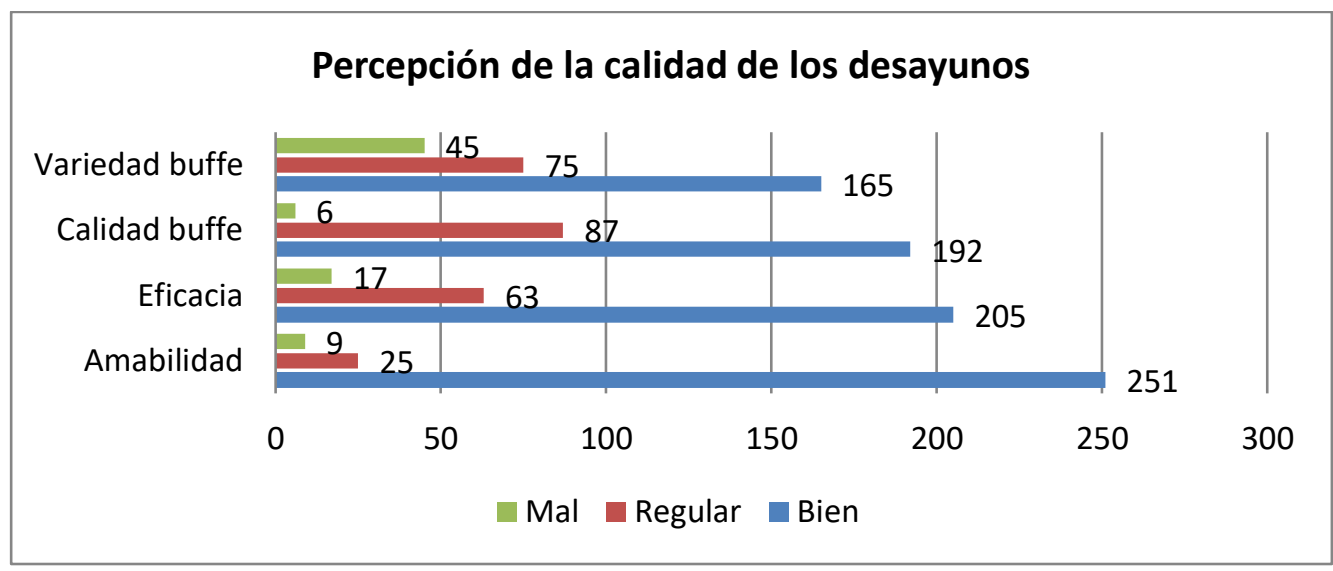

Al analizar las opiniones se aprecia que existe una gran cantidad de encuestados que valoraron de bien los cuatro indicadores seleccionados, destacaron la amabilidad de los empleados, sin embargo, encontraron deficiencias en la calidad del bufé y su variedad, aspectos que afectan directamente el nivel de servicio de restauración de esta área del hotel y que provoca insatisfacciones en los clientes.

Es sabido que los turistas dan un valor muy significativo al desayuno. Este aspecto comienza a ser comprendido por los gerentes, no obstante, todavía no es totalmente adecuado en muchos lugares turísticos de nuestro país. Quizá porque, dada la costumbre de los clientes que nos vistan, es diferente a la de nuestro país, donde el desayuno no recibe la misma consideración que la comida o la cena. Aunque, también, porque es muy común resolver esta primera actividad del día con poco personal de servicio y un bufé estándar. Sin embargo, a consideración de varios autores el desayuno constituye la primera y más importante comida del día.

Figura 2. Percepción sobre la calidad en el servicio de restaurante del "Gran Hotel".

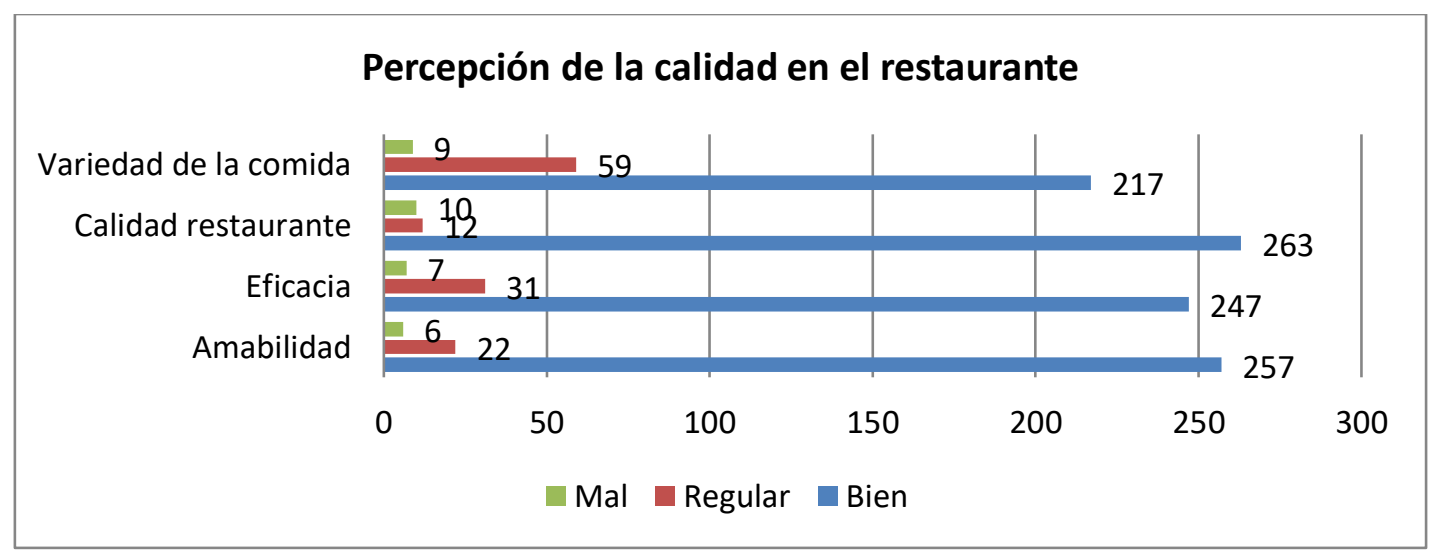


Sobre las relaciones entre las variables de la calidad del servicio en el restaurante, se puede decir que se encontraron relaciones aceptables entre la dimensión de gama tangibles y las tres intangibles. En el caso de la dimensión de aspecto tangible, que se evaluó fue la percepción hacia la variedad de la comida. En lo que refiere a las dimensiones de aspectos intangibles, se valoraron la amabilidad, eficacia y la calidad del restaurante. Así, se evidencia como la falta de variedad en los platos puede ser a veces compensada con una adecuada y esmerada atención, por lo que donde la amabilidad, la cortesía, el respeto pueden atenuar aspectos más tangibles del servicio.

Sin lugar a dudas el restaurante de un hotel es una de las áreas más importantes de una instalación de este tipo, donde además de la comida se vende la cultura culinaria de ese lugar. En muchos casos es el gancho que atrapa a los clientes y los hace volver a ese lugar. Por lo que es un área que no se puede desatender.

Figura 3. Percepción sobre la calidad en el servicio de la cafetería del "Gran Hotel".

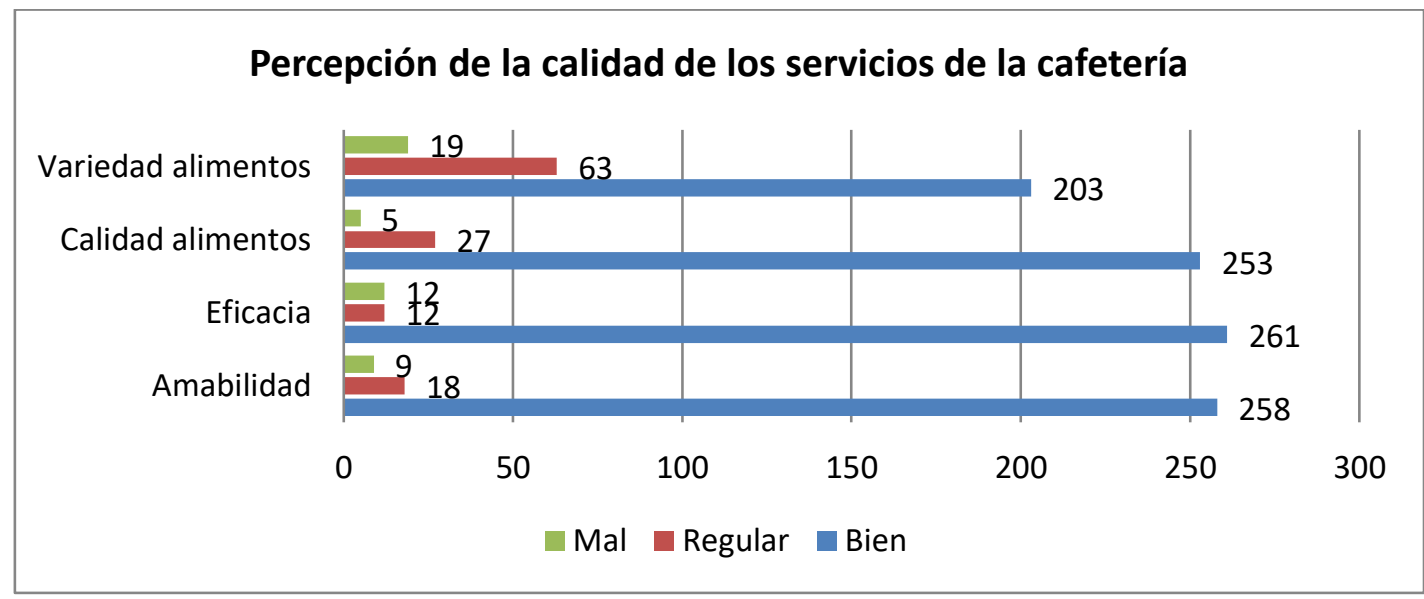

Más del $70 \%$ de los encuestados valoró de bien la calidad del servicio de la cafetería del hotel, el restante rango porcentual lo valoró entre regular y malo. Apreciando que les afecta la poca variedad de la oferta.

Las cafeterías son habituales en cualquier lugar donde existan personas con poco tiempo y deseen degustar un refrigerio, es un servicio rápido a base de alimentos de fácil elaboración y de bebidas alcohólicas y no alcohólicas. Esta área contribuye de esta forma a lograr las metas de atención y ventas establecidas por la gerencia del hotel, sobre todo en los períodos donde baja el nivel de hospedaje. Significa que la cafetería juega un papel decisivo en la 
instalación porque no sólo brinda servicio a los turistas alojados en el hotel, sino además a personas que no tienen reservación, ya que posee una puerta que da a la calle comercial principal de la ciudad.

\subsection{Propuesta de mejora}

En los diferentes instrumentos utilizados se detectaron deficiencias que deben ser atendidas para mejorar la calidad de los servicios del hotel, por lo que se aporta una propuesta de mejora.

Dentro de las deficiencias que afectan la calidad del servicio de restauración y como parte de esta, la inocuidad de los alimentos, se detectaron:

$>$ Insuficiente frecuencia de las capacitaciones a los trabajadores del hotel. En este sentido Villalobos, D. (2017) expresa: .... en el campo de la inocuidad alimentaria, el mal manejo de las prácticas generadoras de contaminaciones en los alimentos podría relacionarse no sólo con la falta de higiene, que acompaña a la propia percepción del riesgo, se ve con frecuencia influenciada por el nivel de familiarización de los sujetos con ésta, así como con la instrucción y capacitación a la que haya sido sometido el manipulador de alimentos.

Señalamientos negativos a la calidad del bufét y su variedad. Insuficiente variedad de platos. Poca variedad de la oferta de alimentos en la cafetería. Son elementos subjetivos relacionados directamente con la calidad de la restauración y que, aunque no producen enfermedades a los clientes deben ser atendidas por la gerencia del hotel, Martín, R. (2009) expone: "Los recursos turísticos son todos los bienes y servicios que por intermedio de la acción del hombre y de los medios con que cuenta, permiten la actividad turística y satisfacen las necesidades de la demanda, que pueden ser de orígenes naturales o creados por los seres humanos (antrópicos)”.

> Los depósitos de residuos destapados. Villacís, J. (2015) señala que uno de los principales focos de contaminación se debe a la basura o manejo de inadecuado de los deschos que puede, entre otros daños, permitir la proliferación vectores de enfermedades. La lucha contra plagas es una de las maneras más importantes para prevenir las enfermedades transmitidas por alimentos (ETA's). 
> Descongelación inadecuada de cárnicos. Villacís, J. (2015) refiere que durante este proceso, los alimentos sufren modificaciones en sus características físicas y químicas: cambios de temperatura, acidez, actividad del agua, atmósfera gaseosa, etc. En algunos casos, estos cambios suponen una aumento en el número de microorganismos o la selección de algunos que se adaptan mejor a las nuevas condiciones creadas. De este modo, la descongelación incorrecta, la cocción inadecuada, el sometimiento del producto acabado a temperaturas incorrectas, la prolongación de tiempos, desde la preparación hasta el servicio de la comida, la limpieza y desinfección deficientes y la manipulación por parte de personal infectado o con malas prácticas de higiene, suelen facilitar el desarrollo de microorganismos, capaces de causar las ETA's.

Alimentos almacenados inadecuadamente durante la congelación. Salvatierra (2019) señala que la conservación de los alimentos o productos orgánicos es importante porque retrasa la alteración estructural, disminuye la presencia de los microorganismos que causan el deterioro, mejora el valor nutritivo y permite la elaboración de más productos alimentarios. Además, al ser productos de consumo directo para el ser humano, su mala preservación puede incidir en la salud de las personas.

> Tabla de corte muy deteriorada. Este aspecto constituye un foco potencial donde pueden proliferar microorganismos. Al respecto Farmacia Lavernia (2018) señala:

Los peligros biológicos en los alimentos se manifiestan en forma de organismos unicelulares que, una vez dentro del cuerpo humano, son capaces de multiplicarse en el interior a modo de incrementar la capacidad infectiva, causando un daño en el huésped.

> Trabajadores con poco dominio de elementos fundamentales que pueden ocasionar conductas que favorecen la perdida de la inocuidad de los alimentos durante su manipulación. Para la Organización de las Naciones Unidas (2017) el personal que manipula los alimentos y las bebidas simbolizan un factor sumamente importante ya que determinarían la inocuidad del producto, son los responsables encargados que deben contar con óptimo estado de salud, mantener una buena higiene personal que implica el adecuado lavado de las 
manos, vestir con una ropa que lo proteja a él y a los alimentos y, sobre todo, crear y practicar hábitos higiénicos de forma constante.

La propuesta de mejora se realiza atendiendo a brindar sugerencias prácticas de acuerdo a los tipos de clientes: internos y externos. Se inicia con la de los clientes internos, que representan un elemento más activo y dinámico de la instalación y con su actuar pueden trasformar los problemas en soluciones que mejoren la satisfacción de los clientes

\subsubsection{Propuesta de cartilla para debate colectivo dentro de las diversas áreas del hotel}

La calidad en el servicio es una disciplina que abarca toda la complejidad de una organización: políticas, formación del personal, relaciones de trabajo, sistemas, opiniones de clientes, diseño del servicio... Y justamente por esta complejidad es que muchos se paralizan, sin saber por dónde empezar.

¿Hay algún modo de mejorar la calidad en el servicio de manera simple en el hotel?

¡Claro! Es más, si aún no hiciste nada concreto por mejorar la calidad de tu servicio, te recomiendo empezar así, sin complicaciones. En esta cartilla se brinda un método que, además de ser simple, es efectivo, y de aplicación inmediata. ¡Esto quiere decir que puedes empezar... ya!

Antes de explicar este método, es necesario aclarar que el proceso de mejorar la calidad del servicio es en realidad algo complejo, ya que están involucrados todos los aspectos organizativos, personales, motivacionales, etc. Pero esto no quita que se pueda comenzar de un modo simple, e ir incorporando poco a poco lo que se vaya necesitando.

Este método es sencillo, pero pone en juego lo esencial de la calidad en el servicio:

$\checkmark \quad$ Un claro enfoque al cliente.

$\checkmark$ La gestión de procesos.

$\checkmark$ El comprometimiento de todo el personal.

$\checkmark$ La toma de decisiones basada en hechos. 
¿Todo esto en un método simple? ¡Sí!

Uno de los clientes está realizando este proceso con resultados muy concretos. Vamos a ejemplificar cada paso del método con esta experiencia real.

PASO 1: Averiguar las principales debilidades del servicio.

Seguro has estado trabajando ya en mejorar el servicio, muy probablemente hayas realizado alguna encuesta de satisfacción a los clientes. O quizás tienes algún otro modo de recibir sus opiniones.

La idea es que captes, de toda esa información, UNA sola debilidad del servicio. La que creas más importante. Pero no se preocupe a la hora de verificar que sea realmente la más importante.

De las opiniones de los clientes se repetían quejas sobre el desayuno.

PASO 2: Evalúa la satisfacción de tus clientes en ese aspecto (sólo ese).

El segundo paso del método es evaluar la satisfacción de los clientes en relación a ese aspecto que se ha identificado. Pero para esto debes hacer una encuesta enfocada.

¿Qué es una encuesta enfocada? Es una encuesta de satisfacción donde sólo preguntas acerca de un aspecto de tu servicio.

¿Qué debes preguntar en esta encuesta?

$\checkmark$ Se explica brevemente al cliente que se está evaluando ese aspecto del servicio para mejorar su satisfacción.

$\checkmark$ El primer punto es una evaluación general (puede ser, por ejemplo, del 1 al 5).

$\checkmark$ Luego se pregunta qué deberá cambiar en ese aspecto para que sea de su total satisfacción (a redactar por el cliente, sin opciones).

En el caso del hotel, se pueden colocar estas breves encuestas en las mesas de desayuno, y se obtendrá una tasa de respuesta muy alta (a diferencia de las encuestas generales que están en las habitaciones).

Es importante colocar las encuestas en momentos y lugares estratégicos. 
PASO 3: Implementar mejoras inmediatamente.

No esperar a tener muchas encuestas respondidas para comenzar a mejorar. Si ves que algo se comienza a repetir, impleméntalo cuanto antes, aunque sea de modo provisional, para evaluar la reacción de los clientes.

En este punto es importante que se involucren los empleados, porque pueden aportar valiosas ideas.

En el hotel, una idea puede ser que la recepción anote en cada encuesta el tipo de cliente del que se trata, para analizar si los requerimientos correspondían a tendencias según el segmento de mercado.

Si un día un huésped indica que desea mayor variedad de mermeladas. Y si ese requerimiento se está repitiendo, al día siguiente impleméntelo.

PASO 4: Evaluar el proceso y consolidar.

Después de tener varias encuestas, debes poner fin a las consultas. Para evaluar todo lo que se hizo y decidir lo que quedará instalado como nueva forma de trabajo. Analizar los costos y beneficios y tomar las decisiones.

Pasado un tiempo de implementados los cambios, se debe volver a realizar la misma encuesta, pero sólo preguntando la evaluación general (el puntaje del 1 al 5). De este modo se podrá confirmar si la satisfacción de los clientes se ha incrementado de manera efectiva.

Nota: Esta cartilla puede ser aplicada por un facilitador del propio hotel o con la ayuda de profesores de la escuela de turismo del territorio. Y se podrán tomar de ejemplo cualquier problema identificado en las áreas, para que los propios trabajadores vean el efecto que produce en su servicio.

Otro aspecto de gran importancia es desarrollar un proceso de capacitación permanente con los empleados que manipulan los alimentos y valorar los aspectos que resultaron negativos en las encuestas aplicadas, para elevar su nivel de conocimientos y que los empleados de mayor experiencia aporten ideas que contribuyan a mejorar el servicio de restauración.

Se decidió, con el pleno apoyo de la gerencia del hotel, desarrollar un plan de capacitación que reforzara las temáticas deficientes, ya que no deja de ser de 
prioridad ubicar en un nivel de conocimiento alto al personal que labora con los alimentos, así como contar con las condiciones y requisitos indispensables para garantizar una excelente calidad sanitaria e inocuidad de los alimentos.

Realizar análisis de los señalamientos de las Inspecciones Sanitarias Estatales para lograr la solución de algunas de las debilidades que constituyen error en los modos de actuar y pueden ser eliminados con facilidad.

\section{CONCLUSIONES}

Mediante este trabajo de investigación se logró determinar el grado de la calidad del servicio de restauración del Hotel "Gran Hotel", arrojando que existen problemas que afectan la calidad de este servicio en esa instalación turista. En lo referente al personal manipulador de alimentos incidieron ciertas prácticas higiénicas no adecuadas y se evidenció una mejoría de la situación higiénicosanitaria de las áreas del hotel a medida que se desarrolló el estudio.

La propuesta de mejoramiento de la calidad del servicio en el Hotel "Gran Hotel" de la ciudad de Camagüey, incluye sugerencias prácticas atendiendo a los clientes: internos y externos. De manera que se puedan satisfacer las necesidades y expectativas de ambos segmentos. Una vez puesta en práctica la propuesta brindará un mejor servicio a los clientes externos, así como mayor imagen de la instalación y mejores ingresos financieros.

\section{REFERENCIAS}

Bergaglio, J. P. 2018. Conceptos clave en la producción de alimentos. Alimentación. Revista Énfasis. Pp 76-78. Año XXIV, N8, septiembre 2018. Buenos Aires, Argentina.

Farmacia, L. (2018). Peligros biológicos de los alimentos. Disponible en: https://farmacialavernia.com/es/peligros-biologicos-de-los- alimentos/

Ferreiro, A. (2019). Manual de Calidad. Higiene e Inocuidad de los Alimentos en el sector del turismo. Santo Domingo, República Dominicana.

NC ISO 22000. (2018). Sistemas de Gestión de la inocuidad de los alimentos. Requisitos para cualquier organización de la cadena alimentaria. 
Kotler, P. (2018). Marketing and production management: Analysis, planning and control, Englewood Cliffs. Prentice Hall.

Niño, M. (2020). Sistema de Gestión de la Calidad en la Industria Alimentaria, herramienta para controlar un problema de salud pública. Universidad Militar Nueva Granada Facultad de Relaciones Internacionales, Estrategia y Seguridad. Bogotá D.C.

Organización Mundial de la Salud (OMS). Organización de Naciones Unidas (ONU) (2017). Enfermedades de transmisión alimentaria. Disponible en: https://www.who.int/topics/foodborne_diseases/es/.

Organización Mundial de la Salud. (2019). Centro de prensa. Organización Mundial de la Salud. Disponible en: https://www.who.int/es/newsroom/fact-sheets/detail/food-safety

Salvatierra, I. (2019). Manual de conservación de alimentos.

Soler Grillo, O., (2017) El turismo en Cuba: economía y estrategia sociopolítica. Ediciones Balcón. La Habana, Cuba. pp. 1 - 8.

Villacís, J. del P. (2015), Diseño y propuesta de un sistema de inocuidad alimentaria basado en BPM para Destino y Hotel de la ciudad de Baños. Trabajo de investigación para optar por el título de Magister en Sistemas de Gestión de Calidad. Instituto de Investigación y Posgrado. Quito. Ecuador. UCE. 402 p. 